\title{
QUALIDADE SANITÁRIA E FISIOLÓGICA DE SEMENTES DE SOJA EM FUNÇÃO DO TRATAMENTO QUÍMICO DE SEMENTES E FOLIAR NO CAMPO
}

\author{
${ }^{\bullet}$ Anderson Luis Danelli ${ }^{1}$, Felipe Rafael Garcés Fiallos ${ }^{1,2,3}$, Rosane Baldiga Tonin ${ }^{1}$, Carlos Alberto Forcelini ${ }^{1}$. \\ ${ }^{1}$ Universidade de Passo Fundo. Laboratório de Fitopatologia, CEP 99052-900, Passo Fundo, RS, Brasil. \\ ${ }^{\circ}$ andersondanelli@hotmail.com, \\ ${ }^{2}$ Unidad de Investigación Cientifica y Tecnológica, Universidad Técnica Estatal de Quevedo, km 7 vía \\ Quevedo - El Empalme, C. P. 73. Mocache, Los Ríos, Ecuador. \\ ${ }^{3}$ Bolsista da Secretaria Nacional de Educação Superior, Ciência e Tecnologia do Equador - SENACYT.
}

\section{Resumo}

$\mathrm{O}$ trabalho teve como objetivo avaliar a qualidade sanitária e fisiológica de sementes de soja em função do tratamento químico de sementes e foliar no campo. Os experimentos foram realizados nos Laboratórios de Fitopatologia e de Sementes da Universidade de Passo Fundo - UPF da Faculdade de Agronomia e Medicina Veterinária - FAMV. As cultivares utilizadas para o trabalho foram FUNDACEP 53 RR e FUNDACEP 55 RR, provenientes do campo experimental da UPF, safra 2009-2010. O trabalho foi composto de 28 tratamentos para cada cultivar, totalizando 56. As amostras das duas cultivares foram submetidos a diferentes combinações de tratamento de sementes, Cropstar ${ }^{\circledR}+$ Derosal plus ${ }^{\circledR}$, Cropstar ${ }^{\circledR}+$ Derosal plus ${ }^{\circledR}+$ Atento ${ }^{\circledR}$, Standak Top ${ }^{\circledR}$ e testemunha (sem aplicação de fungicidas), sendo as doses utilizadas de acordo com as recomendações dos produtos comerciais. As aplicações de fungicidas na parte aérea foram com Opera ${ }^{\circledR}$ (Piraclostrobina+Epoxiconazol), Sphere Max ${ }^{\circledR}$ (Trifloxistrobina+Ciproconazol) nas doses de 150, $500 \mathrm{~mL} \mathrm{ha}^{-1} \mathrm{e}$ testemunha (sem aplicação de fungicidas). $\mathrm{O}$ delineamento experimental foi inteiramente casualizado em todos os experimentos. Foram realizadas avaliações de germinação e sanidade das sementes. Os patógenos que predominaram foram fungos dos gêneros Aspergillus spp. , Penicillium spp. e Alternaria alternata, com os maiores índices para Penicillium spp. e Aspergillus spp. Por outro lado, a qualidade fisiológica das sementes foi preservada, não se observando efeito de fitotoxicidade.

Palavras chaves: Glycine max, fungos, teste de germinação, patologia de sementes, controle químico.
Abstract

$\mathrm{T}$ his study aimed to evaluate the health and physiological quality of soybean seeds due to the chemical treatment of seed and leaf in the field. The experiments were performed in the Laboratories of Plant Pathology and Seeds of the University of Passo Fundo - UPF of College of Agronomy and Veterinary Medicine-FAMV. The cultivars used in this work were FUNDACEP 53 RR and FUNDACEP 55 RR from the research field of the UPF, harvest was in 2009-2010. The work was composed of 28 treatments for each cultivar, 56 in total. The samples of both cultivars were subjected to different combinations of seed treatment, Cropstar ${ }^{\circledR}+$ Derosal plus ${ }^{\circledR}$, Cropstar ${ }^{\circledR}+$ Derosal plus ${ }^{\circledR}$ + Atento ${ }^{\circledR}$, Standak Top ${ }^{\circledR}$ and control (no fungicide aplication), and the doses being used in accordance with the recommendations of the commercial products. The fungicides applications in the aereal part were made with Opera ${ }^{\circledR}$ (Piraclostrobina+Epoxiconazol), Sphere Max ${ }^{\circledR}$ (Trifloxistrobina+Ciproconazol) in doses of 150, $500 \mathrm{~mL} \mathrm{ha}^{-1}$ and control (no fungicide aplication). The experiment design was randomized in all the experiments. The germination and seed health were also evaluated. The pathogens of the genero Aspergillus sp., Penicillium sp. and Alternaria alternata, were the predominant fungi with the highest rates for Penicillium spp. and Aspergillus spp. On the other hand, the physiological seed quality was preserved, with no significant effect of phytotoxicity observed.

Key words: Glycine max, fungi, germination test, seed pathology, chemical control

\section{INTRODUÇão}

$\mathrm{A}$ produção de soja [Glycine $\max$ (L.) Merrill] no Brasil expandiu-se rapidamente no início dos anos

Recibido: 16-Agosto-2011. Recibido en forma corregida: 4-Noviembre2011. Aceptado: 29-Noviembre-2011.

Publicado como NOTA TÉCNICA en Ciencia y Tecnología 4(2):29-37. 2011
70 como uma produção tipicamente agroindustrial. Atingiu um pico em 1989, com 24 milhões de toneladas, 
caindo no início da década 90 (abaixo de 20 milhões ton/ano), recuperando-se progressivamente, até superar a marca de 30 milhões de toneladas na safra 1997-1998.

O Brasil é um dos maiores produtores e exportadores de soja no mundo. Na safra 2008-2009, o Brasil produziu 57.16 milhões de toneladas, em uma área de 21.74 milhões de hectares e com uma produtividade média de 2,629 $\mathrm{kg}$ por hectare (CONAB, 2009).

A disseminação e a transmissão de patógenos em áreas isentas geralmente é realizada por sementes infectadas. O inóculo inicial da epidemia pode depender da transmissão do patógeno pela semente e a presença de patógenos pode também, reduzir a qualidade fisiológica das mesmas. Recomenda-se, portanto, que haja uma integração entre os testes de sanidade e de qualidade fisiológica de sementes (Neergaard, 1977; Menten, 1995). A presença de patógenos após o ponto de maturidade fisiológica ou no armazenamento das sementes é ameaça séria à qualidade das sementes. Segundo Yorinori (1982), elevadas porcentagens de sementes infectadas estão associadas com decréscimo no poder germinativo e menor desenvolvimento da plântula nos seus primeiros estádios. Para LuccaFilho (1995), fungos associados a sementes podem ser responsáveis pela transmissão de doenças para parte aérea e sistema radicular da planta, decréscimo da qualidade fisiológica das sementes e morte de plântulas.

A maturação da semente compreende uma série de alterações morfológicas, fisiológicas e funcionais, que ocorrem a partir da fertilização do óvulo, prosseguindo até o momento em que as sementes estão em condições para a colheita. Durante este processo, verificam-se alterações no peso da matéria seca, teor de umidade, tamanho, germinação e vigor das sementes, ocorrendo, também, alterações na composição química, ou seja, alterações nos teores de carboidratos, proteínas, lipídios, entre outras (Delouche, 1971).

Conforme Carter e Hartwig (1963), a qualidade das sementes de soja é influenciada pela variedade e pelo meio ambiente durante o desenvolvimento e também pelas condições de colheita e armazenamento. A exposição das sementes a períodos de alta umidade após a maturação pode causar danos fisiológicos ou reduzir a sua qualidade (Howell et al., 1959).

Segundo Popinigis (1977), a qualidade fisiológica da semente pode ser definida como a capacidade de desempenhar funções vitais, caracterizada pela germinação, vigor e longevidade, que afeta diretamente a implantação da cultura em condições de campo. A análise de rotina de sanidade de sementes contribui para a avaliação da qualidade de lotes de sementes em culturas de importância econômica, provavelmente devido ao fato de suas sementes transportarem patógenos que podem causar danos à germinação ou quando transmitidas aos órgãos aéreos causar doenças na cultura afetando a produção (Moraes, 1995).

Segundo Dunleavy (1976), as sementes de soja antes da colheita são infectadas por vários tipos de microorganismos, cuja ocorrência tem sido associada a decréscimos na germinação. $\mathrm{O}$ grande número de microorganismos fitopatogênicos pode ser transmitido pelas sementes de soja, sendo o grupo dos fungos o mais numeroso. A ocorrência de fungos em sementes de soja tem sido relatada em diferentes países do mundo onde a cultura é explorada. Até 1981, já haviam sido encontradas 35 espécies de fungos transmitidos pelas sementes dessa leguminosa (Pererira, 2005). Dentre os fungos encontrados em sementes de soja, os de maior ocorrência no Brasil e também de maior importância, causando perdas significativas na produção, são: Phomopsis sp., Sclerotinia sclerotiorum Lib (De Bary), Colletotrichum truncatum Andrus e Moore, Cercospora kikuchii (Matsu. e Tomoy.) Gardner, C. sojina Hara e Perenospora manshurica (Noum). Syd., conforme Zambolim e Chaves (1978). Outros fungos, como Rhizoctonia solani, Kuhn, Fusarium semitectum, Berk. e Rav., Aspergillus spp. e Penicillium spp. são também achados em sementes de soja, podendo causar a sua deterioração e morte de plântulas (Henning et al.,1991). Carter e Hartiwig (1963) afirmam que a maior causa de baixa qualidade de sementes está associada com Phomopsis sojae (Lehman), fato que tem sido confirmado por vários pesquisadores.

A maioria dos patógenos associados às sementes são transmitidos para a parte aérea das plântulas e podem ser visualizados através de sintomas característicos (lesões). Nestas lesões, o patógeno se reproduz e seus propágulos são disseminados e inoculados em tecidos da própria planta e em plantas vizinhas, aumentando a quantidade de doença na área cultivada. Quanto maior a incidência do patógeno nas sementes maior será a porcentagem de focos no campo e mais cedo terá início a epidemia (Menten, 1995).

O objetivo deste estudo foi avaliar a qualidade sanitária e fisiológica de sementes de dois cultivares de soja em função do tratamento químico de sementes e foliar no campo.

\section{Material e Métodos}

$\mathrm{O}$ s experimentos foram realizados nos Laboratórios de Fitopatologia e de Sementes da Universidade de Passo Fundo - UPF da Faculdade de Agronomia e Medicina Veterinária - FAMV. As cultivares utilizadas para o trabalho foram FUNDACEP 53 RR e FUNDACEP $55 \mathrm{RR}$, provenientes do campo experimental da UPF 
da safra 2008-2009. As sementes foram armazenadas em câmara fria $\left(10^{\circ} \mathrm{C}-60 \% \mathrm{UR}\right)$ no Laboratório de Sementes da UPF.

O trabalho foi composto de 28 tratamentos para cada cultivar, totalizando 56. As amostras das duas cultivares foram submetidas a diferentes combinações de tratamento de sementes, Cropstar $\AA+$ Derosal plus ${ }^{\circledR}$,
Cropstar ${ }^{\circledR}+$ Derosal plus $\AA+$ Atento $\AA$, Standak Top $\AA$ e testemunha (sem aplicação de fungicida), para o tratamento químico, as sementes foram pesadas, colocadasemsacosplásticoseentãoaplicadootratamento, agitando as sementes por 5 minutos para melhor contato do produto. As doses utilizadas foram de acordo com as recomendações dos produtos comerciais (Tabela 1).

Tabela 1. Fungicidas aplicados para o controle de doenças na folha e em sementes de soja nas cultivares FUNDACEP 53 RR e FUNDACEP 55 RR, Passo Fundo, RS, Brasil. Safra 2008-2009

\begin{tabular}{|c|c|c|c|c|c|}
\hline \multirow[b]{2}{*}{$\begin{array}{l}\text { Trata- } \\
\text { mentos }\end{array}$} & \multirow[b]{2}{*}{ Cultivares } & \multicolumn{2}{|l|}{ Fungicidas } & \multicolumn{2}{|c|}{ Doses } \\
\hline & & Sementes & Parte aérea & $\begin{array}{l}\text { Sementes } \\
\left(\mathrm{ml} \mathrm{kg}^{-1}\right)\end{array}$ & $\begin{array}{c}\text { Parte aérea } \\
\left(\mathrm{L} \mathrm{ha}^{-1}\right)-\mathrm{VC}^{1}\end{array}$ \\
\hline 1 & FUNDACEP 53 & Testemunha & Testemunha & Não tratada & Não tratada \\
\hline 2 & FUNDACEP 53 & Cropstar ${ }^{\circledR}+$ Derosal plus ${ }^{\circledR}$ & Testemunha & $3+2$ & Não tratada \\
\hline 3 & FUNDACEP 53 & 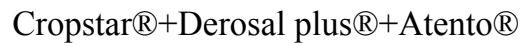 & Testemunha & $3+2+3$ & Não tratada \\
\hline 4 & FUNDACEP 53 & Standak top ${ }^{\circledR}$ & Testemunha & 2 & Não tratada \\
\hline 5 & FUNDACEP 53 & Testemunha & Sphere $\max { }^{\circledR}$ & Não tratada & $150-100$ \\
\hline 6 & FUNDACEP 53 & Cropstar ${ }^{\circledR}+$ Derosal plus ${ }^{\circledR}$ & Sphere $\max { }^{\circledR}$ & $3+2$ & $150-100$ \\
\hline 7 & FUNDACEP 53 & Cropstar ${ }^{\circledR}+$ Derosal plus $\AA+$ Atento $\AA$ & Sphere $\max { }^{\circledR}$ & $3+2+3$ & $150-100$ \\
\hline 8 & FUNDACEP 53 & Standak top ${ }^{\circledR}$ & Sphere $\max { }^{\circledR}$ & 2 & $150-100$ \\
\hline 9 & FUNDACEP 53 & Testemunha & Opera ${ }^{\circledR}$ & Não tratada & $500-100$ \\
\hline 10 & FUNDACEP 53 & Cropstar ${ }^{\circledR}+$ Derosal plus ${ }^{\circledR}$ & Opera $\AA$ & $3+2$ & $500-100$ \\
\hline 11 & FUNDACEP 53 & Cropstar ${ }^{\circledR}+$ Derosal plus ${ }^{\circledR}+$ Atento $\AA$ & Opera ${ }^{\circledR}$ & $3+2+3$ & $500-100$ \\
\hline 12 & FUNDACEP 53 & Standak top ${ }^{\circledR}$ & Opera ${ }^{\circledR}$ & 2 & $500-100$ \\
\hline 13 & FUNDACEP 53 & Testemunha & Sphere $\max { }^{\circledR}$ & Não tratada & $150-150$ \\
\hline 14 & FUNDACEP 53 & Cropstar ${ }^{\circledR}+$ Derosal plus ${ }^{\circledR}$ & Sphere $\max { }^{\circledR}$ & $3+2$ & $150-150$ \\
\hline 15 & FUNDACEP 53 & Cropstar ${ }^{\circledR}+$ Derosal plus $\AA+$ Atento $\AA$ & Sphere $\max { }^{\circledR}$ & $3+2+3$ & $150-150$ \\
\hline 16 & FUNDACEP 53 & Standak top ${ }^{\circledR}$ & Sphere $\max { }^{\circledR}$ & 2 & $150-150$ \\
\hline 17 & FUNDACEP 53 & Testemunha & Opera ${ }^{\circledR}$ & Não tratada & $500-150$ \\
\hline 18 & FUNDACEP 53 & Cropstar ${ }^{\circledR}+$ Derosal plus ${ }^{\circledR}$ & Opera ${ }^{\circledR}$ & $3+2$ & $500-150$ \\
\hline 19 & FUNDACEP 53 & Cropstar ${ }^{\circledR}+$ Derosal plus $\AA+$ Atento $\AA$ & Opera ${ }^{\circledR}$ & $3+2+3$ & $500-150$ \\
\hline 20 & FUNDACEP 53 & Standak top ${ }^{\circledR}$ & Opera ${ }^{\circledR}$ & 2 & $500-150$ \\
\hline 21 & FUNDACEP 53 & Testemunha & Sphere $\max { }^{\circledR}$ & Não tratada & $150-200$ \\
\hline 22 & FUNDACEP 53 & Cropstar ${ }^{\circledR}+$ Derosal plus ${ }^{\circledR}$ & Sphere $\max { }^{\circledR}$ & $3+2$ & $150-200$ \\
\hline 23 & FUNDACEP 53 & Cropstar ${ }^{\circledR}+$ Derosal plus ${ }^{\circledR}+$ Atento $\AA$ & Sphere $\max { }^{\circledR}$ & $3+2+3$ & $150-200$ \\
\hline 24 & FUNDACEP 53 & Standak top ${ }^{\circledR}$ & Sphere $\max { }^{\circledR}$ & 2 & $150-200$ \\
\hline 25 & FUNDACEP 53 & Testemunha & Opera ${ }^{\circledR}$ & Não tratada & $500-200$ \\
\hline 26 & FUNDACEP 53 & Cropstar ${ }^{\circledR}+$ Derosal plus ${ }^{\circledR}$ & Opera ${ }^{\circledR}$ & $3+2$ & $500-200$ \\
\hline 27 & FUNDACEP 53 & Cropstar ${ }^{\circledR}+$ Derosal plus $\AA+$ Atento $\AA$ & Opera ${ }^{\circledR}$ & $3+2+3$ & $500-200$ \\
\hline 28 & FUNDACEP 53 & Standak top $\AA$ & Opera ${ }^{\circledR}$ & 2 & $500-200$ \\
\hline 1 & FUNDACEP 55 & Testemunha & Testemunha & Não tratada & Não tratada \\
\hline 2 & FUNDACEP 55 & Cropstar ${ }^{\circledR}+$ Derosal plus ${ }^{\circledR}$ & Testemunha & $3+2$ & Não tratada \\
\hline
\end{tabular}




\begin{tabular}{|c|c|c|c|c|c|}
\hline 3 & FUNDACEP 55 & Cropstar ${ }^{\circledR}+$ Derosal plus ${ }^{\circledR}+$ Atento ${ }^{\circledR}$ & Testemunha & $3+2+3$ & Não tratada \\
\hline 4 & FUNDACEP 55 & Standak top ${ }^{\circledR}$ & Testemunha & 2 & Não tratada \\
\hline 5 & FUNDACEP 55 & Testemunha & Sphere $\max { }^{\circledR}$ & Não tratada & $150-100$ \\
\hline 6 & FUNDACEP 55 & Cropstar ${ }^{\circledR}+$ Derosal plus ${ }^{\circledR}$ & Sphere $\max { }^{\circledR}$ & $3+2$ & $150-100$ \\
\hline 7 & FUNDACEP 55 & Cropstar ${ }^{\circledR}+$ Derosal plus ${ }^{\circledR}+$ Atento ${ }^{\circledR}$ & Sphere $\max { }^{\circledR}$ & $3+2+3$ & $150-100$ \\
\hline 8 & FUNDACEP 55 & Standak top ${ }^{\circledR}$ & Sphere $\max { }^{\circledR}$ & 2 & $150-100$ \\
\hline 9 & FUNDACEP 55 & Testemunha & Opera ${ }^{\circledR}$ & Não tratada & $500-100$ \\
\hline 10 & FUNDACEP 55 & Cropstar ${ }^{\circledR}+$ Derosal plus ${ }^{\circledR}$ & Opera ${ }^{\circledR}$ & $3+2$ & $500-100$ \\
\hline 11 & FUNDACEP 55 & Cropstar ${ }^{\circledR}+$ Derosal plus ${ }^{\circledR}+$ Atento $(\mathbb{}$ & Opera ${ }^{\circledR}$ & $3+2+3$ & $500-100$ \\
\hline 12 & FUNDACEP 55 & Standak top ${ }^{\circledR}$ & Opera ${ }^{\circledR}$ & 2 & $500-100$ \\
\hline 13 & FUNDACEP 55 & Testemunha & Sphere $\max { }^{\circledR}$ & Não tratada & $150-150$ \\
\hline 14 & FUNDACEP 55 & Cropstar ${ }^{\circledR}+$ Derosal plus ${ }^{\circledR}$ & Sphere $\max { }^{\circledR}$ & $3+2$ & $150-150$ \\
\hline 15 & FUNDACEP 55 & Cropstar ${ }^{\circledR}+$ Derosal plus ${ }^{\circledR}+$ Atento $\AA$ & Sphere $\max { }^{\circledR}$ & $3+2+3$ & $150-150$ \\
\hline 16 & FUNDACEP 55 & Standak top ${ }^{\circledR}$ & Sphere $\max { }^{\circledR}$ & 2 & $150-150$ \\
\hline 17 & FUNDACEP 55 & Testemunha & Opera ${ }^{\circledR}$ & Não tratada & $500-150$ \\
\hline 18 & FUNDACEP 55 & Cropstar ${ }^{\circledR}+$ Derosal plus ${ }^{\circledR}$ & Opera ${ }^{\circledR}$ & $3+2$ & $500-150$ \\
\hline 19 & FUNDACEP 55 & Cropstar ${ }^{\circledR}+$ Derosal plus ${ }^{\circledR}+$ Atento $\AA$ & Opera ${ }^{\circledR}$ & $3+2+3$ & $500-150$ \\
\hline 20 & FUNDACEP 55 & Standak top ${ }^{\circledR}$ & Opera ${ }^{\circledR}$ & 2 & $500-150$ \\
\hline 21 & FUNDACEP 55 & Testemunha & Sphere $\max { }^{\circledR}$ & Não tratada & $150-200$ \\
\hline 22 & FUNDACEP 55 & Cropstar ${ }^{\circledR}+$ Derosal plus ${ }^{\circledR}$ & Sphere $\max { }^{\circledR}$ & $3+2$ & $150-200$ \\
\hline 23 & FUNDACEP 55 & Cropstar ${ }^{\circledR}+$ Derosal plus ${ }^{\circledR}+$ Atento $\AA$ & Sphere $\max { }^{\circledR}$ & $3+2+3$ & $150-200$ \\
\hline 24 & FUNDACEP 55 & Standak top ${ }^{\circledR}$ & Sphere $\max { }^{\circledR}$ & 2 & $150-200$ \\
\hline 25 & FUNDACEP 55 & Testemunha & Opera ${ }^{\circledR}$ & Não tratada & $500-200$ \\
\hline 26 & FUNDACEP 55 & Cropstar ${ }^{\circledR}+$ Derosal plus ${ }^{\circledR}$ & Opera ${ }^{\circledR}$ & $3+2$ & $500-200$ \\
\hline 27 & FUNDACEP 55 & Cropstar ${ }^{\circledR}+$ Derosal plus ${ }^{\circledR}+$ Atento $\AA$ & Opera ${ }^{\circledR}$ & $3+2+3$ & $500-200$ \\
\hline 28 & FUNDACEP 55 & Standak top ${ }^{\circledR}$ & Opera ${ }^{\circledR}$ & 2 & $500-200$ \\
\hline
\end{tabular}

${ }^{1} \mathrm{VC}$ : Volume de calda expressada em litros.

Após o tratamento das sementes ocorreu à semeadura a campo. As aplicações de fungicidas na parte aérea foram com Opera ${ }^{\circledR}$ (Piraclostrobina+Epoxiconazol), Sphere Max ${ }^{\circledR} \quad$ (Trifloxistrobina + Ciproconazol) nas doses de $150,500 \mathrm{~mL} \mathrm{ha}^{-1}$ e testemunha (sem aplicação de fungicida) (Tabela 1). Foram realizadas três aplicações dos fungicidas mencionados cada 21 dias de intervalo de tempo.

Após a colheita das parcelas, foi realizado o teste de sanidade e germinação das sementes colhidas, nos Laboratórios de Fitopatologia e Sementes, respectivamente.

$\mathrm{Na}$ condução do teste de sanidade, foi empregado o método de plaqueamento das sementes em meio de cultura BSA+antibiótico (estreptomicina).

As sementes foram esterilizadas em uma solução de hipoclorito de sódio+água destilada (1:1) por 5 minutos, após colocadas em caixas de acrílico tipo gerbox. Em cada caixa foram plaqueadas 25 sementes, totalizando 400 para cada tratamento.

Transferiu-se para uma câmara de crescimento com controle de temperatura de $25^{\circ} \mathrm{C}$ e fotoperíodo de 12 horas. A avaliação da incidência dos fungos presentes em sementes foi realizada sete dias após a instalação do experimento com auxílio de microscópio estereoscópico e ótico marca ZEISS modelo Stemi 2000-C, sendo os resultados expressos em porcentagem de incidência de fungos.

O teste de germinação foi realizado com 400 sementes (quatro sub-amostras de 100 sementes) para cada cultivar. As sementes foram semeadas em rolos de papel germitest, com quantidade de água equivalente a 2.5 vezes o seu peso e colocadas em germinador regulado a $25^{\circ} \mathrm{C}$, por cinco dias, segundo critérios adotados por Brasil (1992).

$\mathrm{O}$ delineamento experimental foi inteiramente casualizado em todos os experimentos. Os dados foram submetidos à análise de variância e, realizou-se 
Tabela 2. Incidência de fungos em sementes de soja, cultivares FUNDACEP 53 RR e FUNDACEP 55 RR, expressos em porcentagem de sementes infectadas, Passo Fundo, RS, Brasil. Safra 2008-2009

\begin{tabular}{|c|c|c|c|c|c|c|c|c|c|c|c|c|}
\hline \multirow[t]{2}{*}{ Trat. $^{1}$} & \multicolumn{2}{|c|}{$\begin{array}{l}\text { Alternaria } \\
\text { alternata }\end{array}$} & \multicolumn{2}{|c|}{$\begin{array}{l}\text { Aspergillus } \\
\text { spp. }\end{array}$} & \multicolumn{2}{|c|}{$\begin{array}{c}\text { Cercospora } \\
\text { spp. }\end{array}$} & \multicolumn{2}{|c|}{$\begin{array}{l}\text { Fusarium } \\
\text { semitectum }\end{array}$} & \multicolumn{2}{|c|}{$\begin{array}{l}\text { Penicillum } \\
\text { spp. }\end{array}$} & \multicolumn{2}{|c|}{$\begin{array}{c}\text { Colletotrichum } \\
\text { truncatum }\end{array}$} \\
\hline & F. $5^{2}$ & F. $55^{3}$ & F. 53 & F. 55 & F. 53 & F. 55 & F. 53 & F. 55 & F. 53 & F. 55 & F. 53 & F. 55 \\
\hline 1 & $3.88 \mathrm{abc}^{6}$ & $2.81 \mathrm{ab}$ & $1.97 \mathrm{~b}$ & $1.06 \mathrm{ab}$ & $1.06 \mathrm{~b}$ & $0.71 \mathrm{~b}$ & $0.71 \mathrm{a}$ & $0.71 \mathrm{a}$ & $0.71 \mathrm{c}$ & $3.43 \mathrm{a}$ & $0.71 \mathrm{a}$ & 1.0 \\
\hline 2 & $4.46 \mathrm{abc}$ & $2.37 \mathrm{ab}$ & $5.77 \mathrm{a}$ & $1.97 \mathrm{ab}$ & $0.71 \mathrm{~b}$ & $0.71 \mathrm{~b}$ & $1.90 \mathrm{a}$ & $1.06 \mathrm{a}$ & $0.71 \mathrm{c}$ & $2.38 \mathrm{a}$ & $0.71 \mathrm{a}$ & $0.71 \mathrm{~b}$ \\
\hline 3 & $2.12 \mathrm{c}$ & $2.83 \mathrm{ab}$ & $0.71 \mathrm{~b}$ & $3.54 \mathrm{a}$ & $0.71 \mathrm{~b}$ & $0.71 \mathrm{~b}$ & $1.06 \mathrm{a}$ & $1.26 \mathrm{a}$ & $5.52 \mathrm{ab}$ & $3.21 \mathrm{a}$ & $0.71 \mathrm{a}$ & $0.71 \mathrm{~b}$ \\
\hline 4 & $3.31 \mathrm{abc}$ & $1.66 \mathrm{ab}$ & $2.55 \mathrm{~b}$ & $1.41 \mathrm{ab}$ & $0.71 \mathrm{~b}$ & $0.71 \mathrm{~b}$ & $0.71 \mathrm{a}$ & $1.77 \mathrm{a}$ & $2.12 \mathrm{bc}$ & $1.41 \mathrm{a}$ & $0.71 \mathrm{a}$ & $0.71 \mathrm{~b}$ \\
\hline 5 & $3.63 \mathrm{abc}$ & $1.06 \mathrm{ab}$ & $1.06 \mathrm{~b}$ & $1.77 \mathrm{ab}$ & $0.71 \mathrm{~b}$ & $1.06 \mathrm{~b}$ & $1.06 \mathrm{a}$ & $1.26 \mathrm{a}$ & $6.94 \mathrm{a}$ & $2.83 \mathrm{a}$ & $0.71 \mathrm{a}$ & $0.71 \mathrm{~b}$ \\
\hline 6 & $3.10 \mathrm{abc}$ & $2.12 \mathrm{ab}$ & $3.20 \mathrm{ab}$ & $1.61 \mathrm{ab}$ & $0.71 \mathrm{~b}$ & $0.71 \mathrm{~b}$ & $1.06 \mathrm{a}$ & $0.71 \mathrm{a}$ & $7.07 \mathrm{a}$ & $4.21 \mathrm{a}$ & $0.71 \mathrm{a}$ & $0.71 \mathrm{~b}$ \\
\hline 7 & $4.72 \mathrm{abc}$ & $2.52 \mathrm{ab}$ & $1.61 \mathrm{~b}$ & $0.71 \mathrm{~b}$ & $0.71 \mathrm{~b}$ & $1.26 \mathrm{ab}$ & $0.71 \mathrm{a}$ & $1.66 \mathrm{a}$ & $7.01 \mathrm{a}$ & $3.18 \mathrm{a}$ & $1.06 \mathrm{a}$ & $0.71 \mathrm{~b}$ \\
\hline 8 & $3.58 \mathrm{abc}$ & $1.61 \mathrm{ab}$ & $1.26 \mathrm{~b}$ & $1.26 \mathrm{ab}$ & $0.71 \mathrm{~b}$ & $1.06 \mathrm{~b}$ & $0.71 \mathrm{a}$ & $0.71 \mathrm{a}$ & $5.80 \mathrm{ab}$ & $3.31 \mathrm{a}$ & $0.71 \mathrm{a}$ & $0.71 \mathrm{~b}$ \\
\hline 9 & $2.16 \mathrm{c}$ & $0.71 \mathrm{~b}$ & $1.61 \mathrm{~b}$ & $1.41 \mathrm{ab}$ & $0.71 \mathrm{~b}$ & $1.06 \mathrm{~b}$ & $1.06 \mathrm{a}$ & $0.71 \mathrm{a}$ & $7.44 \mathrm{a}$ & $2.36 \mathrm{a}$ & $0.71 \mathrm{a}$ & $0.71 \mathrm{~b}$ \\
\hline 10 & $2.52 \mathrm{bc}$ & $1.41 \mathrm{ab}$ & $1.06 \mathrm{~b}$ & $0.71 \mathrm{~b}$ & $0.71 \mathrm{~b}$ & $1.06 \mathrm{ab}$ & $0.71 \mathrm{a}$ & $1.06 \mathrm{a}$ & $5.09 \mathrm{abc}$ & $2.47 \mathrm{a}$ & $0.71 \mathrm{a}$ & $0.71 \mathrm{~b}$ \\
\hline 11 & $3.92 \mathrm{abc}$ & $1.81 \mathrm{ab}$ & $2.12 \mathrm{~b}$ & $2.12 \mathrm{ab}$ & $0.71 \mathrm{~b}$ & $1.41 \mathrm{ab}$ & $0.71 \mathrm{a}$ & $1.06 \mathrm{a}$ & $3.21 \mathrm{abc}$ & $4.08 \mathrm{a}$ & $1.06 \mathrm{a}$ & $0.71 \mathrm{~b}$ \\
\hline 12 & $2.92 \mathrm{abc}$ & $1.61 \mathrm{ab}$ & $0.71 \mathrm{~b}$ & $0.71 \mathrm{~b}$ & $0.71 \mathrm{~b}$ & $1.61 \mathrm{~b}$ & $0.71 \mathrm{a}$ & $0.71 \mathrm{a}$ & $3.72 a b c$ & $3.08 \mathrm{a}$ & $0.71 \mathrm{a}$ & $0.71 \mathrm{~b}$ \\
\hline 13 & $2.83 \mathrm{abc}$ & $1.06 \mathrm{ab}$ & $1.41 \mathrm{~b}$ & $1.77 \mathrm{ab}$ & $1.06 \mathrm{ab}$ & $0.71 \mathrm{~b}$ & $1.41 \mathrm{a}$ & $1.06 \mathrm{a}$ & $3.88 \mathrm{abc}$ & $3.58 \mathrm{a}$ & $1.41 \mathrm{a}$ & $1.41 \mathrm{ab}$ \\
\hline 14 & $2.47 \mathrm{bc}$ & $2.72 \mathrm{ab}$ & $1.77 \mathrm{~b}$ & $1.06 \mathrm{ab}$ & $0.71 \mathrm{~b}$ & $0.71 \mathrm{~b}$ & $1.06 \mathrm{a}$ & $0.71 \mathrm{a}$ & $4.98 \mathrm{abc}$ & $3.31 \mathrm{a}$ & $0.71 \mathrm{a}$ & $0.71 \mathrm{~b}$ \\
\hline 15 & $3.78 \mathrm{abc}$ & $1.90 \mathrm{ab}$ & $1.06 \mathrm{~b}$ & $2.12 \mathrm{ab}$ & $1.06 \mathrm{ab}$ & $0.71 \mathrm{~b}$ & $0.71 \mathrm{a}$ & $0.71 \mathrm{a}$ & $6.76 \mathrm{ab}$ & $2.83 \mathrm{a}$ & $1.97 \mathrm{a}$ & $1.06 \mathrm{ab}$ \\
\hline 16 & $97 \mathrm{c}$ & $3.93 \mathrm{ab}$ & $0.71 \mathrm{~b}$ & $1.61 \mathrm{ab}$ & $0.71 \mathrm{~b}$ & $0.71 \mathrm{~b}$ & $1.41 \mathrm{a}$ & $1 \mathrm{a}$ & $3.63 \mathrm{abc}$ & $3.07 \mathrm{a}$ & $0.71 \mathrm{a}$ & $0.1 \mathrm{~b}$ \\
\hline 17 & $3.73 \mathrm{abc}$ & 3.0 & $1 \mathrm{~b}$ & $b$ & $\mathrm{~b}$ & & $1.41 \mathrm{a}$ & & $6.15 \mathrm{ab}$ & $0 \mathrm{a}$ & $2 \mathrm{a}$ & $a b$ \\
\hline 18 & $3.67 \mathrm{abc}$ & 2.3 & $\mathrm{~b}$ & 1.0 & $b$ & $0.71 \mathrm{~b}$ & 1.4 & $\mathrm{a}$ & $6.32 \mathrm{ab}$ & $6 a$ & $2 a$ & $\mathrm{~b}$ \\
\hline 19 & $3.00 \mathrm{abc}$ & $1.26 \mathrm{ab}$ & $1.06 \mathrm{~b}$ & $2.10 \mathrm{ab}$ & $1.26 \mathrm{ab}$ & $2.72 \mathrm{a}$ & $1.81 \mathrm{a}$ & $\mathrm{a}$ & $6.43 \mathrm{ab}$ & $3.51 \mathrm{a}$ & $1.41 \mathrm{a}$ & $5 a b$ \\
\hline 20 & $3.64 \mathrm{abc}$ & $1.06 \mathrm{ab}$ & $0.71 \mathrm{~b}$ & $1.06 \mathrm{ab}$ & $0.71 \mathrm{~b}$ & $1.26 \mathrm{ab}$ & $1.06 \mathrm{a}$ & $0.71 \mathrm{a}$ & $5.53 \mathrm{ab}$ & $1.06 \mathrm{a}$ & $1.77 \mathrm{a}$ & $0.71 \mathrm{~b}$ \\
\hline 21 & $5.60 \mathrm{a}$ & $2.92 \mathrm{ab}$ & $1.66 \mathrm{~b}$ & $2.45 \mathrm{ab}$ & $0.71 \mathrm{~b}$ & $1.06 \mathrm{~b}$ & $1.41 \mathrm{a}$ & $0.71 \mathrm{a}$ & $5.32 \mathrm{ab}$ & $3.61 \mathrm{a}$ & $2.12 \mathrm{a}$ & $0.71 \mathrm{~b}$ \\
\hline 22 & $3.93 \mathrm{abc}$ & $4.08 \mathrm{a}$ & $0.71 \mathrm{~b}$ & $2.45 \mathrm{ab}$ & $0.71 \mathrm{~b}$ & $1.06 \mathrm{~b}$ & $0.71 \mathrm{a}$ & $0.71 \mathrm{a}$ & $6.08 \mathrm{ab}$ & $2.52 \mathrm{a}$ & $1.41 \mathrm{a}$ & $2.16 \mathrm{a}$ \\
\hline 23 & $5.21 \mathrm{abc}$ & $1.26 \mathrm{ab}$ & $0.71 \mathrm{~b}$ & $0.71 \mathrm{~b}$ & $1.26 \mathrm{ab}$ & $1.06 \mathrm{~b}$ & $1.06 \mathrm{a}$ & $0.71 \mathrm{a}$ & $7.37 \mathrm{a}$ & $2.96 \mathrm{a}$ & $1.61 \mathrm{a}$ & $0.71 \mathrm{~b}$ \\
\hline 24 & $3.00 \mathrm{ab}$ & $3.63 \mathrm{ab}$ & $0.71 \mathrm{~b}$ & $0.71 \mathrm{~b}$ & $0.71 \mathrm{~b}$ & $0.71 \mathrm{~b}$ & $1.06 \mathrm{a}$ & $1.97 \mathrm{a}$ & $5.72 \mathrm{ab}$ & $3.03 \mathrm{a}$ & $1.97 \mathrm{a}$ & $1.06 \mathrm{ab}$ \\
\hline 25 & $3.00 \mathrm{abc}$ & $1.41 \mathrm{ab}$ & $2.92 \mathrm{ab}$ & $2.32 \mathrm{ab}$ & $1.81 \mathrm{ab}$ & $1.06 \mathrm{~b}$ & $1.41 \mathrm{a}$ & $0.71 \mathrm{a}$ & $4.03 \mathrm{abc}$ & $1.77 \mathrm{a}$ & $1.06 \mathrm{a}$ & $0.71 \mathrm{~b}$ \\
\hline 26 & $3.64 \mathrm{abc}$ & $3.16 \mathrm{ab}$ & $1.26 \mathrm{~b}$ & $1.17 \mathrm{ab}$ & $1.81 \mathrm{ab}$ & $0.71 \mathrm{~b}$ & $0.71 \mathrm{a}$ & $0.71 \mathrm{a}$ & $3.61 \mathrm{abc}$ & & $1.97 \mathrm{a}$ & $1.06 \mathrm{ab}$ \\
\hline 27 & $5.23 \mathrm{ab}$ & $2.72 \mathrm{ab}$ & $1.06 \mathrm{~b}$ & $0.71 \mathrm{~b}$ & $1.41 \mathrm{ab}$ & $1.06 \mathrm{ab}$ & $0.71 \mathrm{a}$ & $0.71 \mathrm{a}$ & $6.01 \mathrm{ab}$ & $1.77 \mathrm{a}$ & $1.97 \mathrm{a}$ & $1.26 \mathrm{ab}$ \\
\hline 28 & $3.38 \mathrm{abc}$ & $2.12 \mathrm{ab}$ & $1.90 \mathrm{~b}$ & $2.61 \mathrm{ab}$ & $2.36 \mathrm{a}$ & $2.25 \mathrm{ab}$ & $0.71 \mathrm{a}$ & $0.71 \mathrm{a}$ & $4.49 \mathrm{abc}$ & $2.52 \mathrm{a}$ & $1.81 \mathrm{a}$ & $1.06 \mathrm{ab}$ \\
\hline $\mathrm{CV}(\%)^{4}$ & 30.01 & 45.11 & 67.00 & 53.39 & 50.82 & 48.72 & 59.58 & 60.32 & 31.21 & 32.82 & 42.61 & 48.4 \\
\hline Sig. ${ }^{5}$ & $* *$ & $* *$ & $* *$ & $* *$ & $* *$ & $* *$ & NS & NS & $* *$ & NS & NS & $* *$ \\
\hline & $\begin{array}{l}3 \\
5\end{array}$ & . & & & & & & & & & & \\
\hline
\end{tabular}


comparação das médias através do teste de Tukey, ao nível de 5\% de probabilidade. Embora, para a diferença estatística entre os cultivares foi realizado o teste de F.

\section{Resultados e Discussão}

$\mathrm{O}$ resultados de incidência de fungos em sementes obtidos em função do emprego dos diferentes tratamentos para as cultivares FUNDACEP $53 \mathrm{RR}$ e FUNDACEP 55 RR constam na tabela 2.

Com base nos dados obtidos na avaliação da sanidade das sementes de soja colhidas, submetidas aos diferentes tratamentos em sementes, aplicações foliares e doses do produto, os fungos identificados nas amostras analisadas foram: Fusarium semitectum, Alternaria alternata, Cercospora spp, Colletotrichum truncatum, Aspergillus spp. e Penicillium spp. Na figura 1 pode- se observar que ouve diferenças entre os tratamentos, e na Tabela 2 nota-se diferenças estatísticas significativas entre cada uno dos tratamentos avaliados na maioria dos fungos, não existindo para $F$. semitectum e $C$. truncatum, esta última somente na cultivar FUNDACEP 53 RR. Em trabalhos conduzidos por Wink et al. (1985) e Tronbeta e Wink (1987), no Rio Grande do Sul, demonstraram que os fungos patogênicos mais importantes encontrados associados a sementes de soja foram Fusarium spp. (predominância de F. semitectum), Cercospora kikuchii, C. sojina, Colletotrichum truncatum, Phomopsis sp. e Rhizoctonia solani. Também Pereira et al. (2005) relata que este grupo de patógenos são de importância econômica (Fusarium spp., Cercospora kikuchii, Colletotrichum truncatum e Aspergillus spp), e outros de importância secundária, porém destacados com bastante freqüência (Alternaria spp. e Penicillium spp.).

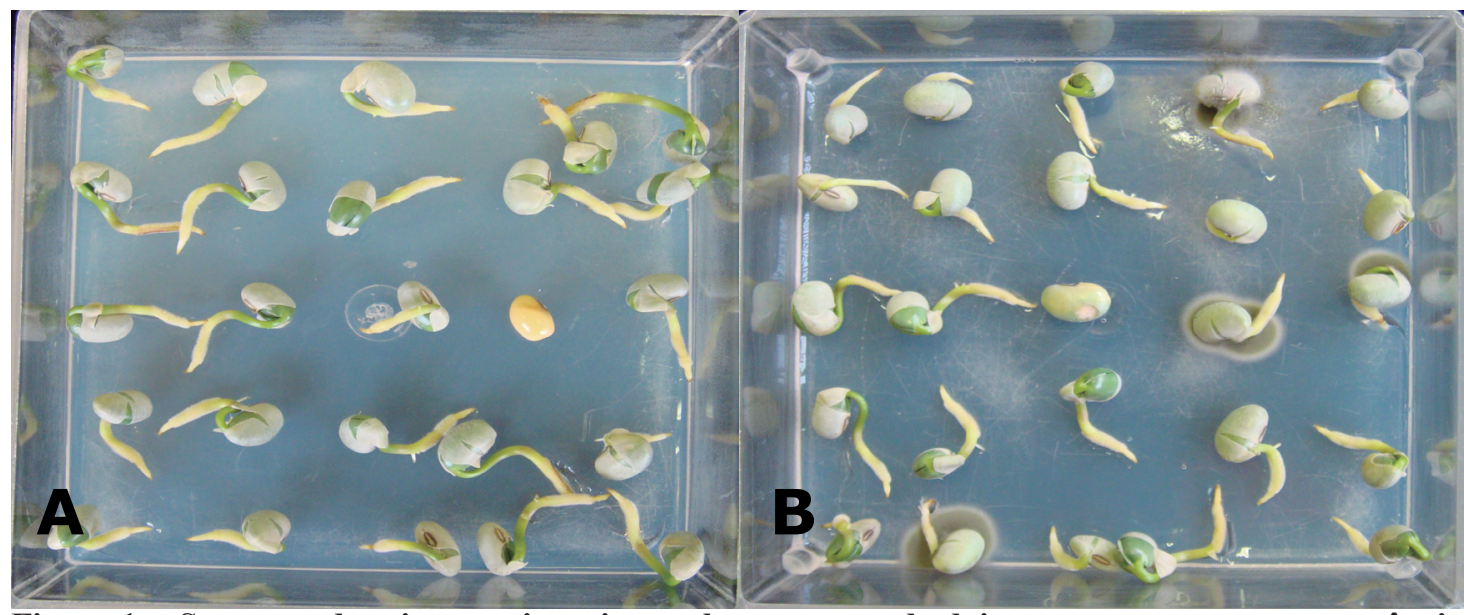

Figura 1. Sementes de soja em caixas tipo gerbox, mostrando dois tratamentos: um com ausência (A) e outro com presencia de fungos (B).

Goulart et al. (1995) relata que em amostras de sementes de soja analisadas na safra 1992-1993, sendo estas, provenientes de diversos locais do Estado de Mato Grosso, foram identificados 23 gêneros de fungos, sendo os encontrados com maior freqüência Fusarium semitectum, seguido de Aspergillus sp., Penicillium sp., Phomopsis sp., Cercospora kikuchii, Cladosporium sp., Colletotrichum truncatum e Alternaria alternata. De acordo com o mesmo autor, variações na incidência de fungos associados a sementes são observadas em função do local de produção e/ou das condições climáticas.

Conforme mostra a tabela 2, para a cultivar FUNDACEP $53 \mathrm{RR}$, os valores de incidência de Alternaria alternata variaram de 1.97 à $5.60 \%$, Aspergillus spp. de 0.71 à $5.77 \%$, Cercospora spp. de 0.71 à $2.36 \%$, Fusarium semitectum de 0.71 à $1.41 \%$, Penicillium spp. de 0.71 à $7.37 \%$ e Colletotrichum truncatum de 0.71 à $2.12 \%$, respectivamente. Os fungos de armazenamento (Aspergillus spp. e Penicillium spp.) apresentaram incidência relativamente elevada nas sementes analisadas. Choudhury (1987) e Lucca-Filho (1995) afirmam que os danos causados pelas espécies de Aspergillus e Penicillium são variáveis, como: perda de germinação, descoloração das sementes, aumento da taxa de ácidos graxos, aquecimento da massa de sementes e produção de toxinas.

No caso da cultivar FUNDACEP 55 RR, os valores de incidência de Alternaria alternata situaramse entre 0.71 à $4.08 \%$, Aspergillus spp. variou de 0.71 à $3.54 \%$, Cercospora spp. 0.71 à $2.72 \%$, Fusarium semitectum de 0.71 à $1.97 \%$, Penicillium spp. de 1.06 à $4.08 \%$ e Colletotrichum truncatum variou de 0.10 à $2.16 \%$. Esses resultados estão de acordo com aqueles obtidos por Bolkan et al. (1976), Zambolin e Chaves (1978), Goulart (1984), Wetzel e Didonet (1984), Wink et al. (1985), Tronbeta e Wink (1987) e Henning et al. (1991), os quais também demonstraram que esses fungos são os de maior ocorrência em sementes de soja 
no Brasil.

Conforme resultados obtidos no teste de sanidade para as sementes de soja, pode se observar ainda que não houve diferença estatística entre os tratamentos para os patógenos: Fusarium semitectum e Colletotrichum truncatum para a cultivar FUNDACEP 53, bem como para Fusarium semitectum e Penicillium spp., para a cultivar FUNDACEP 55 (Tabela 2), indicando que os fungicidas empregados não influenciaram na infecção das sementes por esses patógenos.

Por outro lado, diferença significativa entre os tratamentos foi observada para a cultivar FUNDACEP 53 RR em Alternaria alternata, Aspergillus spp., Cercospora spp. e Penicillium spp., e para a FUNDACEP 55 RR em Alternaria alternata, Aspergillus spp., Cercospora spp. e Colletotrichum truncatum (Tabela 2).

Com relação ao fungo Alternaria alternata, melhores resultados foram alcançados com os tratamentos: Cropstar $\AA+$ Derosal plus $\AA+$ Atento $\AA$ (semente), com dosagem de $3+2+3\left(\mathrm{~mL} \mathrm{~kg}^{-1}\right)$; Opera ${ }^{\circledR}$ (parte aérea) na dosagem de 500 - 100 (L $\mathrm{ha}^{-1}$ ) e Standak top $\AA$ (semente), na dose de 2 (mL $\mathrm{kg}^{-1}$ ), Sphere Max (parte aérea) na dosagem de 150 - 150 (L ha $\left.{ }^{-1}\right)$. Maior incidência desse fungo foi encontrada no tratamento onde foi utilizado Sphere $\operatorname{Max}{ }^{\circledR}$ (parte aérea), na dosagem de 150 - 200 (L $\mathrm{ha}^{-1}$ ), mostrando baixa eficiência do produto.

Para a cultivar FUNDACEP 53 RR, Aspergillus spp., valores elevados de incidência foram encontrados utilizando o tratamento Cropstar $($ +Derosal plus $\AA$ (semente) na dose de $3+2\left(\mathrm{~mL} \mathrm{~kg}^{-1}\right)$ e parte aérea não tratada. Baixa eficiência foi observada também para os fungos Cercospora sp. quando usado Standak top ${ }^{\circledR}$ (semente) na dose de $2\left(\mathrm{~mL} \mathrm{~kg}^{-1}\right)$ e Opera ${ }^{\circledR}$ (parte aérea), na dose de 500 - $200\left(\mathrm{~L} \mathrm{ha}^{-1}\right)$ e Penicillium spp., utilizando Opera ${ }^{\circledR}$ (parte aérea), na dose de 500 - 200 ( $\mathrm{L} \mathrm{ha}^{-1}$ ) (Tabela 2).

Conforme mostra a tabela 2, verificase baixa infecção na cultivar FUNDACEP 55 RR para Alternaria alternata quando do emprego de Opera ${ }^{\circledR}$ (parte aérea), na dose de 500 - $100\left(\mathrm{~L} \mathrm{ha}^{-1}\right)$, já para Aspergillus spp. o uso de Cropstar ${ }^{\circledR}+$ Derosal plus ${ }^{\circledR}+$ Atento $\AA$ (semente) na dose de $3+2+3$ $\left(\mathrm{mL} \mathrm{kg} \mathrm{kg}^{-1}\right.$, resultou em baixa eficiência, o mesmo se observando para Cercospora sp., quando usado Cropstar $\AA+$ Derosal plus $\AA+$ Atento $\AA$ (semente) na dose de $3+2+3\left(\mathrm{~mL} \mathrm{~kg}^{-1}\right)$, Opera $\AA$ (parte aérea), na dose de 500 - $150\left(\mathrm{~L} \mathrm{ha}^{-1}\right)$, e, Cropstar ${ }^{\circledR}+$ Derosal plus ${ }^{\circledR}$ (semente) na dose de $3+2\left(\mathrm{~mL} \mathrm{~kg}^{-1}\right)$, Sphere $\operatorname{Max}{ }^{\circledR}$ (parte aérea), na dose de 150 - $200\left(\mathrm{~L} \mathrm{ha}^{-1}\right)$, para Colletotrichum truncatum.

A qualidade das sementes, avaliada pelo teste
Tabela 3. Porcentagem de germinação de sementes de soja, cultivares FUNDACEP 53 RR e FUNDACEP 55 RR, Passo Fundo, RS, Brasil. Safra 2008-2009

\begin{tabular}{|c|c|c|}
\hline \multirow{2}{*}{ Tratamentos } & \multicolumn{2}{|c|}{ Porcentagem de germinação } \\
\hline & F. $53^{1}$ & F. $5^{2}$ \\
\hline 1 & $87.50 \mathrm{bc}^{5}$ & 87.50 cdef \\
\hline 2 & $91.00 \mathrm{abc}$ & $87.50 \mathrm{cdef}$ \\
\hline 3 & $89.50 \mathrm{abc}$ & $93.00 \mathrm{abcd}$ \\
\hline 4 & $88.00 \mathrm{abc}$ & $93.00 \mathrm{abcd}$ \\
\hline 5 & $87.00 \mathrm{bc}$ & $85.50 \mathrm{f}$ \\
\hline 6 & $87.50 \mathrm{bc}$ & 91.00 abcdef \\
\hline 7 & $90.50 \mathrm{abc}$ & $93.50 \mathrm{abc}$ \\
\hline 8 & $91.00 \mathrm{abc}$ & 91.50 abcdef \\
\hline 9 & $91.00 \mathrm{abc}$ & 90.00 abcdef \\
\hline 10 & $94.00 \mathrm{a}$ & 92.50 abcde \\
\hline 11 & $93.00 \mathrm{ab}$ & $95.00 \mathrm{ab}$ \\
\hline 12 & $93.00 \mathrm{ab}$ & $96.00 \mathrm{a}$ \\
\hline 13 & $88.00 \mathrm{abc}$ & 90.00 abcdef \\
\hline 14 & $93.00 \mathrm{ab}$ & 89.00 bcdef \\
\hline 15 & $91.50 \mathrm{abc}$ & 90.00 abcdef \\
\hline 16 & $91.50 \mathrm{abc}$ & 90.00 abcdef \\
\hline 17 & $91.50 \mathrm{abc}$ & 86.00 ef \\
\hline 18 & $91.00 \mathrm{abc}$ & $93.50 \mathrm{abc}$ \\
\hline 19 & $90.00 \mathrm{abc}$ & $94.50 \mathrm{ab}$ \\
\hline 20 & $90.00 \mathrm{abc}$ & 92.00 abcdef \\
\hline 21 & $92.50 \mathrm{ab}$ & 89.50 abcdef \\
\hline 22 & $92.00 \mathrm{abc}$ & 90.00 abcdef \\
\hline 23 & $94.00 \mathrm{a}$ & $93.50 \mathrm{abc}$ \\
\hline 24 & $94.00 \mathrm{a}$ & $93.00 \mathrm{abcd}$ \\
\hline 25 & $87.00 \mathrm{bc}$ & $86.50 \mathrm{def}$ \\
\hline 26 & $93.00 \mathrm{ab}$ & 87.50 cdef \\
\hline 27 & $86.00 \mathrm{c}$ & $96.00 \mathrm{a}$ \\
\hline 28 & $88.00 \mathrm{abc}$ & $93.50 \mathrm{abc}$ \\
\hline $\operatorname{CV}(\%)^{3}$ & 1.75 & 2.79 \\
\hline Sig. ${ }^{4}$ & $* *$ & $* *$ \\
\hline
\end{tabular}

${ }^{1}$ FUNDACEP 53

${ }^{2}$ FUNDACEP 55

${ }^{3}$ Coeficiente de variação

${ }^{4}$ Significância estadística pelo teste de F: ** (altamente significativo)

${ }^{5}$ Médias seguidas pela mesma letra na coluna não diferem estatisticamente pelo teste de Tukey a 5\% de probabilidade. 
de germinação, apresentou diferenças significativas entre os tratamentos nas duas cultivares. Valores médios obtidos na germinação de sementes de soja, variaram de 86 a 94\%, para a cultivar FUNDACEP 53 RR e para a cultivar FUNDACEP 55 RR entre 85.50 e 96.0\%, respectivamente (Tabela 3 ).

Os resultados mostrados neste trabalho são similares à pesquisa realizada por Garcés et al. (2009), que avaliando a germinação e o vigor de sementes de soja, altura de plantas e ocorrência de fungos na cultivar FUNDACEP 55 RR, encontrou um melhor desempenho dos fungicidas Derosal plus ${ }^{\circledR}+$ Atento ${ }^{\circledR}$ e Maxim $\mathrm{XL} \circledast$, pois eles proporcionaram melhor emergência de plântulas a 13 e $23^{\circ} \mathrm{C}$. Por outro lado, houve diferenças significativas para germinação, altura de planta e incidência de doenças (Fusarium verticillioides, Alternaria spp. e Pythium spp., Fusarium verticillioides, Pythium spp., Rhizoctonia spp.e Thielaviopsis basicola, além de uma bactéria não identificada).

Características superiores de sementes foram observadas na cultivar FUNDACEP 53 RR, quando as mesmas foram submetidas aos tratamentos Cropstar ${ }^{\circledR}+$ Derosal plus ${ }^{\circledR}$ (semente) e Opera ${ }^{\circledR}$ (parte aérea), com dosagem de 500 - $100\left(\mathrm{~L} \mathrm{ha}^{-1}\right)$; Cropstar $(+$ Derosal plus ${ }^{\circledR}+$ Atento ${ }^{\circledR} \quad$ (semente) e Sphere Max ${ }^{\circledR}$ (parte aérea), com dosagem de 150 - $200\left(\mathrm{~L} \mathrm{ha}^{-1}\right)$ e Standak top ${ }^{\circledR}$ (semente) e Sphere Max ${ }^{\circledR}$ (parte aérea) com dosagem de $150-200\left(\mathrm{~L} \mathrm{ha}^{-1}\right)$.

Por outro lado, resultados satisfatórios na germinação de sementes de soja na cultivar FUNDACEP 53 RR, foram obtidos com os tratamentos Standak top $\AA$ (semente) e Opera ${ }^{\circledR}$ (parte aérea), na dosagem de 500 - $100\left(\mathrm{~L} \mathrm{ha}^{-1}\right)$ e Cropstar $\AA+D e r o s a l$ plus $\AA+$ Atento $\AA$ (semente) e Opera ${ }^{\circledR}$ (parte aérea), na dosagem de 500 - $200\left(\mathrm{~L} \mathrm{ha}^{-1}\right)$. Nota-se que todos os tratamentos apresentaram porcentagens médias de germinação superiores a mínima estabelecida para a comercialização de sementes de soja, ou seja, $80 \%$ de germinação (MAPA, 2005).

\section{Conclusões}

$\mathrm{O}$ patógenos que predominaram foram fungos dos gêneros Aspergillus spp., Penicillium spp. e Alternaria alternata, com os maiores índices para Penicillium spp. e Aspergillus spp.

Evidencia-se efeito dos fungicidas aplicados em parte aérea e nas sementes de soja, na incidência de fungos e na qualidade fisiológica das sementes nas condições que foi realizado o experimento.

A qualidade fisiológica das sementes foi preservada, não se observando efeito de fitotoxicidade.

\section{LITERATURA CITADA}

Bolkan, H. A., A. R. Silva and F. P. Cupertino. 1976. Fungi associated with soybean and bean seeds and their control in Central Brazil. Plant Disease Reporter 60(6) 454-458.

BRASIL. 1992. Ministério da Agricultura e Reforma Agrária. Secretaria Nacional de Defesa Agropecuária. Departamento Nacional de Produção Vegetal. Coordenação de Laboratório Vegetal. Regras para Análise de Sementes. Brasília, DF. 365 p.

Carter, J. L. and E. E. Hartwig. 1963. The management of soybeans. In: Norman, A. G. The Soybean. New York: Academic Press. p. 162-221

Choudhury, M. M. 1987. Testes de sanidade de sementes de caupi. Em: Soave, Y. e Wetzel, M. M. V. da S. Patologia de Sementes. Campinas: Fundação Cargill. p. 371-385

CONAB (Companhia Nacional de Abastecimento, BR). 2009. Safras. (em line). Consultado 5 jan. 2010. Disponível em www.conab.gov.com

Delouche, J. C. 1971. Seed maturation. In: Handbook of Seed Technology. Mississipi: Mississipi State University.

Dunleavy, J. M. 1976. Pathological factors affecting seed germination In: Hill, L. D. World soybean research. Dauville. p. 462-469

Garcés, F. R., M. C. Ferreira e C. A. Forcelini. 2009. Ocorrência de patógenos e crescimento inicial da soja em função do tratamento químico de sementes e da temperatura de incubação. Informe Abrates. (Edição especial). 405:592. (Resumo).

Goulart, A. C. P. 1984. Avaliação do nível de ocorrência e efeitos de Phomopsis sp. e Sclerotinia sclerotiorum (Lib.) de Bary em sementes de soja (Glycine $\max$ (L.) Merrill). Teses apresentada para a obtenção de Mestre em Agronomia. Escola Superior de Agricultura de Lavras, Lavras. ESAL Brasil. 80 p.

Goulart, A. C. P., F. A. Paiva e P. J. M. Andrade. 1995. Qualidade sanitária de sementes de soja (Glycine $\max (\mathrm{L}$.$) Merrill) produzidas no Mato Grosso do$ Sul. Revista Brasileira de Sementes. 17(1):42-46.

Henning, A. A., F. C. Krzyzanowski, J. B. França Neto e J. T. Yorinori. 1991. Tratamento de sementes de soja com fungicidas. Londrina: EMBRAPACNPSo. (Comunicado Técnico, 49).

Howell, R. W., F. I. Collins and V. E. Sedwick. 1959. Respiration of soybean seeds as related to weathering losses during ripening. Agr. Jour. 51:677-679.

Lucca-Filho, O. A. 1995. Curso de Tecnologia de Sementes. Brasília: ABEAS. 53 p. 
MAPA. Ministério da Agricultura, Pecuária e Abastecimento. 2005. (em line). Consultado 15 Out. 2009. Disponível em http://extranet. agricultura.gov.br/sislegis-consulta/ consultarLegislacao.do?Operação=

Menten, J. O. M. 1995. Patógenos em Sementes: detecção, danos e controle químico. São Paulo: CibaAgro. 321 p.

Moraes, M. H. D. 1995. Testes de sanidade de sementes em rotina no Brasil: situação atual, contribuições e perspectivas. Em: Menten, J. O. M. Patógenos em Sementes: detecção, danos e controle químico. São Paulo: Ciba Agro. p. 37-51.

Neergaard, P. 1977. Seed Pathology. (Vol. 2). London: McMillan Press.

Pererira, G.A.C. 2005. Fungos em sementes de soja. Dourados: EMBRAPA.

Popinigis, F. 1977. Fisiologia da Semente. Brasília: AGIPLAN. 289 p.
Tronbeta, I. A. e R. F. Wink. 1987. Identificação da flora fúngica de sementes de soja (Glycine max (L.) Merrill). ABRATES (Congresso). 183. (Resumo).

Wetzel, M. M. V. da S. e H. R. Didonet. 1984. Qualidade fisiológica e sanitária de sementes de soja em Barra do Garça, MT. Fitopatologia Brasileira 9(2):379. (Resumo).

Wink, R. F., M. R. Oliveira, A. P. Almeida, I. A. Tronbeta e Z. I. Antoniolli. 1985. Identificação da flora fúngica de sementes de soja (Glycine max (L.) Merrill). ABRATES (Congresso). 135. (Resumo).

Yorinori, J. T. 1982. Doenças da Soja Causadas por fungos. Informe Agropecuário. 8:40-46.

Zambolim, L. e G. M. Chaves. 1978. Doenças da soja. Informe Agropecuário. 4(3):38-48. 